\title{
Thyroid Dysfunction in Patients with Liver Disease
}

\author{
R P Buden ${ }^{1}$ \\ ${ }^{1}$ M.D. Gen Medicine, Assistant Professor, Dept of Gen Medicine, SSIMS Medical College, T Begur NelamangalaTq, Bengaluru Rural Dist.
}

\section{Abstract}

Background: Liver disease related to alcohol (Ethanol, ethylalcohol) is very common global problem and is one of the major medical complications of alcohol abuse. Daily consumption is usual for causation of liver disease. Aim: To estimate the prevalence of thyroid dysfunction in patients with liver disease. Subjects and Methods: Early morning fasting serum thyroid stimulating hormone (TSH), serum total free thyroxine (FT4) and free triiodothyronine (FT3) was measured by radioimmunoassays in 100 index patients with liver cirrhosis who did not have history of thyroid diseases. Study Period: November 2018 to October 2019 The age of study population was in the range of 25 65 years. Out of 100 patients, 75 were males and 25 were females. Conclusion: Abnormalities in circulating thyroid hormone concentrations i.e hypothyroidism is noted especially in those with ethanol related liver cirrhosis and it is associated with more advanced liver disease.

Keywords: Liver cirrhosis, Hypothyroidism, Ethanol related liver disease, Hepatitis B related liver disease, CTP score.

Corresponding Author: Dr. R P Buden, M.D. Gen Medicine, Assistant Professor, Dept of Gen Medicine, SSIMS Medical College, T Begur Nelamangala Tq, Bengaluru Rural Dist.

Received: November 2019

Accepted: November 2019

\section{Introduction}

The liver is one of the largest and Cirrhosis and alcoholic hepatitis often of ALD, and ALD can be difficult to most complex organs in the body. coexist and cause substantial morbidity diagnose because patients frequently It performs multiple functions, and mortality. For example, studies from minimize or deny alcohol abuse. In including the production of proteins the Department of Veterans Affairs (VA) addition, there may be no evidence of and enzymes, detoxification, metabolic demonstrate that patients with both cir-ALD from the physical exam, and lab-functions, and the regulation of choles-rhosis and alcoholic hepatitis have a oratory abnormalities may not specifiterol and blood clotting. Because the death rate of greater than 60 percent cally point to ALD. liver is primarily responsible for alcohol over a 4year period, with most of the Ambulatory patients with alcoholic metabolism, it is especially vulnerable deaths occurring in the first year (Chedid fatty liver often are asymptomatic. to alcohol-related injury. et al. 1991). Thus, the mortality rate for Patients with alcoholic hepatitis may be Alcoholic liver disease (ALD) is a ALD is greater than that of many com-asymptomatic, have only enlarged liver serious and potentially fatal consequence mon types of cancer such as colon, breast, (i.e., hepatomegaly), or have full-blown of drinking alcohol. ALD encompasses and prostate. This article examines the three conditions: fatty liver, alcoholic issues of diagnosing and treating ALD LUIS S. MARSANO, M.D., is a professor of hepatitis, and cirrhosis .Fatty and the complications of this disease. internal medicine; CHRISTIAN MENDEZ, liver (i.e., steatosis), the most common M.D., is a gastroenterology fellow; DANIELL alcohol-induced liver disorder, is marked HILL, M.D., is an associate professor of by the excessive accumulation of fat Diagnosis of Alcoholic internal medicine; SHIRISH BARVE, PH.D., inside the liver cells. Alcoholic hepatitis Liver Disease (ALD) is an associate professor of internal medicine; is inflammation and more severe injury and CRAIG J. MCCLAIN, M.D., is vice of the liver, in which the body's immune The diagnosis of ALD is established by chair for research in the Department of system responds to and causes liver a history of habitual alcohol intake of Internal Medicine and professor of inter-damage. In cirrhosis, normal liver cells sufficient duration and quantity, together nal medicine and pharmacology and are replaced by scar tissue (i.e., fibrosis), with physical signs and laboratory evitoxicology; all authors are associated with and consequently the liver is unable to dence of liver disease. Alcohol dependence the University of Louisville Medical perform many of its usual functions. is not a prerequisite for the development Center, Louisville, Kentucky. Alcoholic liver disease effects most of the organs in our body. On this alcoholic liver diseases various studies have been done .It is observed that not many Indian studies on thyroid hormone functional studies in case of alcoholic liver diseases are available. The study of thyroid hormone function tests will throw a light on the functional aspects of liver diseases and gives some better understanding of the alcoholic liver disease and their interrelationship with thyroid function and thus helps in the management of alcoholic liver diseases.

The liver, a plays an important role in thyroid hormone metabolism, being involved in the conjugation and deiodination of thyroxine (T4) and tri-iodothyronine (T3) as 
well as synthesis and secretion of major thyroid hormone binding proteins, thyroxine-binding globulin (TBG), thyroxine-binding prealbumin and albumin.

A large group of alcoholic liver disease patients were studied for the endocrine changes and these changes were correlated to the severity of liver diseases in order to examine the underlying mechanism accounting for endocrine dysfunction. In the majority of patients examined marked changes were found in circulating thyroid hormones T3 below the normal level in the absence of significant changes in TSH. Total T4 was normal or low. They were clinically euthyroid.

In patients with alcoholic cirrhosis the levels of serum T4, T3, reverse T3 (rT3) were determined. The results showed Serum T4, T3 levels were decreased and serum rT3 levels increased in patients with alcoholic cirrhosis. Serum T4 and T3 were lower and rT3 higher in cirrhotic patients who died within three months of study compared with those who survived. It suggests that assay of serum thyroid hormone levels may be helpful in assessing the course and prognosis of patients with liver disease. ${ }^{[3]}$

In alcoholic cirrhosis with clinically euthyroid patients the serum concentrations of thyroid hormones showed the mean serum Total T4 was low and the mean serum total T3 was significantly lower than the normal. The mean serum TSH is increased.

\section{Aims and Objectives}

The aims of this study were:

Primary - To study thyroid hormone level (FT3, FT4, and $\mathrm{TSH})$ in the liver cirrhosis patient.

Secondary - To find out the significance of thyroid hormone level and severity of cirrhosis of the liver.

\section{Study DESIGN}

Prospective cross sectional observational study.

\section{Inclusion Criteria}

Patients with symptoms, signs, biochemical and radiological features of liver cirrhosis irrespective of cause.

\section{Exclusion Criteria}

Liver Disease patients on treatment already and withany complications likehepatic encephalopathy, spontaneous bacterial peritonitis, hepatorenal syndrome, gastrointestinal bleed and patients on thyroid medications already.

\section{Subjects and Methods}

Test subjects are male patients of alcoholic liver disease from This was a hospital-based prospective observational study, conducted in a tertiary care center of South India, SSIMS Medical College, T Begur NelamangalaTq from November 2018 to October 2019. Patients having no history of diseases like diabetes mellitus, hypertension, non alcoholic liver diseases, renal diseases, thyroid disorders.

Control subjects are healthy males who are hospital employees of the hospital. With no current/lifetime history of alcohol drinking

All these subjects were subjected to medical examination as per a fixed proform. Their blood pressure, height, weight, $\mathrm{BMI}$ is recorded in both groups.
Biochemical Tests

Thyroid Function Tests (Elisa Method)

Serum T3

Serum T4

Serum TSH

Liver Function Tests (Routine methods using Auto Analyzers)

1. Total bilirubin -

Direct

Indirect

2. Total protein

Serum albumin

A:G ratio

3. Serum AST

Serum ALT

Serum ALP

Serum GGT.

\section{Results}

A total of 100 liver cirrhosis cases ( 75 males and 25 females) and 100 apparently healthy controls ( 75 male and 25 female) were included in the final analysis. The mean age was $43 \pm$ 14 years for cases and $42 \pm 15$ years for controls. All cases were decompensated liver cirrhosis. During study, gross ascites (74\%) was found most common presentation and other complications were anemia $(87 \%)$, thrombocytopenia $(53 \%)$, coagulation abnormality $(65 \%)$, HE (38\%), jaundice $(32 \%)$, upper gastrointestinal bleed $(34 \%)$, azotemia $(17 \%)$, pleural effusion (16\%), sepsis (22\%), shock (14\%). Constipation was found in $49 \%$ patients. Alcohol was found most common etiology (46\%) followed by hepatitis B (19\%), hepatitis C (3\%), Wilson disease (1\%), and others.

\section{Discussion}

The most common abnormality seen was low FT3 syndrome $41 \%$ (41 out of 100) in cases, $50 \%$ (19 out of 38) in cirrhosis with $\mathrm{HE}$, and $32 \%$ (5 out of 16 ) in nonsurvivor group of liver cirrhosis cases. Hypothyroidism (high TSH level) was observed in $20 \%$ (20 out of 100) of decompensated liver cirrhosis patients, $26.3 \%$ (10 out of 38) in decompensated liver cirrhosis with $\mathrm{HE}$, and $50 \%$ (8 out 16) in nonsurvivor cases. Nonthyroidal illness with low T4 was observed in $15 \%$ (15 out of 100) of decompensated liver cirrhosis patients, $16 \%$ (6 out of 38 ) in decompensated liver cirrhosis with HE, and $12 \%$ (2 out 16) in nonsurvivor group. All cirrhosis patients did not have clinical signs of hypothyroidism and their TSH levels were also in the subclinical range of hypothyroidism. When we looked for individual parameter of thyroid function, the most common abnormality seen was low free T3 level (71\%), low free T4 (21\%), and high TSH level (20\%). Mobin et al. reported that in all decompensated cirrhotic patients (sample size $n=76$ ), $76.3 \%$ (our study $71 \%$ ) had low serum T3 levels, $14.47 \%$ (our study 21\%) had low serum T4 levels, and $2.63 \%$ (our study $20 \%$ ) had raised TSH levels. The results of our study for FT3 levels and FT4 levels are consistent with Mobin et al study, but contradict for TSH levels. This difference may be due to sample size ,age ,sex ,severity of liver disease and 
regional variation of thyroid disorders.

In several studies, the most common abnormalities of serum thyroid hormone concentration in cirrhotic patients observed were, low serum T3 level, raised rT3 level, and a normal TSH levels. There are many factors those may be responsible for these abnormalities, which includes alteration in plasma level of thyroid binding proteins, altered binding of T4 and T3 to their carrier protein, impaired hepatic clearance of reverse T3 (rT3), hyperglucagonemia, and reduced extrathyroidal conversion of T4 to T3. In cirrhotic patients, because of extensive hepatic inflammation and fibrosis, there is inhibition of Type 1 (D1) deiodinase enzymes that lead to decreased conversion of T4 to T3. Since the type 2 (D2) deiodinase enzymes remain active, now most of the T4 is converted into rT3 leading to increased rT3 levels.

Increased level of TSH also statistically significant in cirrhosis nonsurvivors compared to cirrhosis survivors (Subgroup 2) and HE nonsurvivors compared to $\mathrm{HE}$ survivors (Subgroup 3) liver cirrhosis patients. Joeimon et al. reported similar results, but Kayacetin et al. observed no significant difference in TSH Level in liver cirrhosis patients.

\section{Conclusion}

The mean FT3 and FT4 levels were found to be significantly decreased and the mean TSH levels were significantly increased in liver cirrhosis cases compared to healthy controls. Low levels of FT3 also correlated with the severity of liver disease in the form of CTP or MELD. Level of FT4 decreases as CTP Class (A-C) increases. Level of TSH increases with MELD score. Therefore, thyroid levels in cirrhotic patients may be used as a prognostic marker. Low FT3 might be used as a predictor of patients for underlying HE, while low FT3 and high TSH might be used as a predictor of mortality in liver cirrhosis patients, although death in cirrhotic patient is multifactorial.

Abnormalities in circulating thyroid hormone concentrations i.e hypothyroidism is noted especially in those with ethanol related liver cirrhosis and it is associated with more advanced liver disease.

\section{Impact on Society}

Thyroid function test should be done regularly in liver disease patients to reduce morbidity and mortality.

\section{References}

1. P. Burra, J.A. Franklyn, D.B.Ramsden, E.Elias and M.C.Sheppard .Severity of alcoholic liver disease and markers of thyroid and steroid status Post graduate medical journal 1992 october;68(804-810).

2. Y Israel, PG Walfish, H Orrego, J Blake, H Kalant. Thyroid hormone levels in alcoholic liver diseases. Gastroenterology Vol.76, issue 1, January 1979; 116-122

3. Hepner GW, Chopra IJ .Serum thyroid hormone levels in patients with liver disease. Archive Internal Medicine .1979 Oct; 139(10); 1117-20.

4. S Nomura, C S Pittman, J B Chambers, Jr, M W Buck and T Shimizu. .Reduced peripheral conversion of thyroxine to triiodothyronine in patients with hepatic cirrhosis. The Journal of Clinical investigation 1975 September; 56(3) 643-652.

5. JanniA, etal. The prognostic value of thyroid function tests in liver cirrhosis. In Langer M Chiandussi L, Chopra I J and Martin L,eds, The Endocrines and The Liver, London, Academic Press, 1982; 232-6

6. L'age M, Meinhold $\mathrm{H}$, Wenzel $\mathrm{KW}$,Schlesinger $\mathrm{H}$. Relations between serum levels of TSH,TBG,T4,T3,rT3 and various histological classified chronic liver diseases. Journal of Endocrinology investigations 1980 oct-dec; 3(4);379-83.

7. George C. Schussler, M.D.,Fenton Schaffner, M.D., and Felice korn M.S.Increased serum thyroid hormone binding and decreased free hormone in chronic liver disease. The New England Journal of Medicine 1978; 299:510- 515.

8. Williams Text book of Endocrinology Edition 11.Henry M. Krokenberg, Shlomo Melmed Kenneth S.Polonsky, and P.Reed Larsen.

9. M Borzio, R Caldara, F Borzio, V Piepoli et al. Thyroid function tests in chronic liver disease: evidence for multiple abnormalities despite clinical euthyroidism. GUT, an International Journal of Gastroenterology and Hepatology; 1983 July; 24(7); 631-636.

10. Oxford Text book of Clinical hepatology; Vol_2.

Copyright: () the author(s), 2019. It is an open-access article distributed under the terms of the Creative Commons Attribution License (CC BY 4.0), which permits authors to retain ownership of the copyright for their content, and allow anyone to download, reuse, reprint, modify, distribute and/or copy the content as long as the original authors and source are cited.

How to cite this article: Buden RP. Thyroid Dysfunction in Patients with Liver Disease. Acad. J Med. 2019;2(2):138-40.

DOI: dx.doi.org/10.21276/ajm.2019.2.2.35

Source of Support: Nil, Conflict of Interest: None declared. 